\title{
Erratum to: Robot-assisted and conventional therapies produce distinct rehabilitative trends in stroke survivors
}

Francisco J. Valero-Cuevas ${ }^{1,2^{*}}$, Verena Klamroth-Marganska ${ }^{3}$, Carolee J. Winstein ${ }^{2}$ and Robert Riener ${ }^{3}$

\section{Erratum}

The original article [1] contains an error in the Conclusions section.

The original article states the following:

"This alternative approach to, and interpretation of, the results of RCTs will lead to more effective therapies targeted for the multidimensional mechanisms of recovery."

This should instead say:

"This alternative approach to, and interpretation of, the results of RCTs may lead to more effective therapies targeted for the multidimensional mechanisms of recovery."

\section{Author details}

'Department of Biomedical Engineering, University of Southern California, 3710 McClintock Ave, RTH 404, Los Angeles, CA 90089-2905, USA. ²Division of Biokinesiology and Physical Therapy, University of Southern California, Los Angeles, CA, USA. ${ }^{3}$ ETH Zurich and University of Zurich, Zurich, Switzerland.

Received: 29 November 2016 Accepted: 29 November 2016

Published online: 06 December 2016

\section{Reference}

1. Valero-Cuevas FJ, et al. Robot-assisted and conventional therapies produce distinct rehabilitative trends in stroke survivors. J NeuroEngineering Rehabil. 2016;13:92. doi:10.1186/s12984-016-0199-5.

\footnotetext{
* Correspondence: valero@usc.edu

'Department of Biomedical Engineering, University of Southern California,

3710 McClintock Ave, RTH 404, Los Angeles, CA 90089-2905, USA

${ }^{2}$ Division of Biokinesiology and Physical Therapy, University of Southern

California, Los Angeles, CA, USA

Full list of author information is available at the end of the article
} 